\title{
Light curve modelling of close eclipsing binaries
}

\author{
M. Fedurco, M. Čokina and Š. Parimucha
}

Department of Theoretical Physics and Astrophysics, Institute of Physics, Faculty of Natural Sciences, P. J. Śafárik University, Jesenná 5, 040 01, Košice, Slovakia, (E-mail: miroslav.fedurco@student.upjs.sk)

Received: October 28, 2019; Accepted: January 16, 2020

\begin{abstract}
We present an easy to use software package fully written in Python designed for light curve modelling of eclipsing close binaries. The software provides full treatment of the Roche geometry and irradiation effects utilizing symmetries of tidally deformed stellar surfaces and eccentric orbits in order to reduce overall computational time. Additionally, the software package allows modelling of spots and low-amplitude radial and non-radial pulsations.
\end{abstract}

Key words: binaries - computational astrophysics - stars: pulsations

\section{Introduction}

Research on low-amplitude stellar oscillations of eclipsing binary components requires a robust numerical model capable of dealing with phenomena such as pulsations of deformed stellar surfaces, amplitude variation of modes during eclipses, and tidal locking of pulsation modes. Therefore, we present a package ${ }^{1}$ that utilizes a modular approach in which every functionality is treated as a separate object that ensures easy addition of new features.

\section{Capabilities of the package}

Among the capabilities of the presented package are modelling of surfaces of single and binary systems utilizing surface symmetries in order to reduce overall computational time (Fedurco et al., 2019). This package also allows the modelling of binary systems with eccentric orbits, where the surface geometry changes with orbital phase. However, symmetry of component surfaces for orbital positions placed symmetrically around the apsidal line enabled us to greatly reduce the number of required re-calculations of component surfaces.

Using external atmospheric models (Castelli \& Kurucz, 2004) and limb darkening models (van Hamme, 1993) we are able to generate light curves of binary systems for a variety of commonly used bands and instruments.

With such model in place we are able to model surface inhomogeneities such as pulsations and spots. Current capabilities of the package include modelling of low-amplitude oscillations represented by spherical harmonics with an

${ }^{1}$ github.com/mikecokina/elisa 

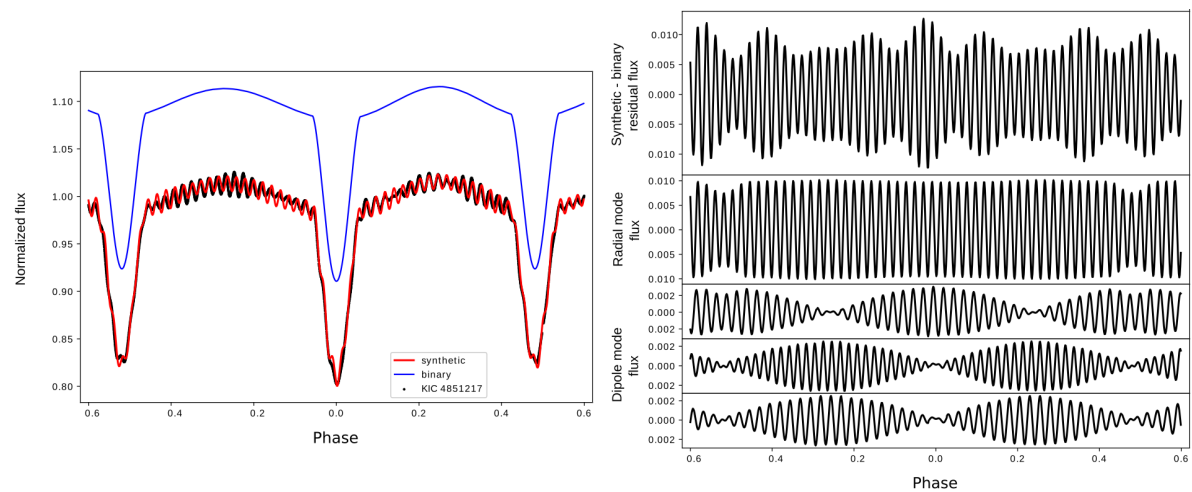

Figure 1. The left panel shows the light curve of the oscillating eclipsing binary KIC 4851217 overlayed with its fitted light curve in red. Variations due to binarity are indicated with a blue line. The right panel shows variations caused by oscillations decomposed to their constituent modes.

option of mode axis misalignment, and modelling of temperature spots on assynchronously rotating components.

\section{Conclusions}

Future versions of this package will support modelling of light curves of single star systems and also will be capable of producing radial velocity curves with full implementation of the Rossiter-McLaughlin effect and effects of pulsations on radial velocity curves.

Acknowledgements. This research was supported by the Slovak Research and Development Agency under the contract No. APVV-15-0458.

\section{References}

Castelli, F. \& Kurucz, R. L. 2004, New Grids of ATLAS9 Model Atmospheres

Fedurco, M., Čokina, M., \& Parimucha, Š., Modelling of stellar surfaces in single and binary star systems. 2019, Contributions of the Astronomical Observatory Skalnate Pleso, 49, 346

van Hamme, W., New Limb-Darkening Coefficients for Modeling Binary Star Light Curves. 1993, Astron. J., 106, 2096, DOI: 10.1086/116788 